\title{
Turbulent Supersonic Channel Flow: Direct Numerical Simulation and Modeling
}

\author{
Stefan Heinz* \\ University of Wyoming, Laramie, Wyoming 82071 \\ DOI: $10.2514 / 1.22259$
}

\begin{abstract}
In this paper, direct numerical simulation results of turbulent supersonic channel flow are analyzed to address several questions that are relevant to turbulence modeling: the turbulence frequency production mechanism, wall damping effects on turbulence model parameters, and the relevance of compressibility effects. Limited support is found for usually applied models for the turbulence frequency production and wall damping effects. In contrast to that it is shown that turbulence frequency production mechanisms and wall damping effects may be explained very well on the basis of a frequency scaling that characterizes mean flow changes. The influence of compressibility is found to be relevant.
\end{abstract}

\section{Nomenclature}

$a \quad=$ mean speed of sound, $(\gamma R \bar{T})^{1 / 2}$

$a_{w} \quad=$ mean speed of sound at the wall, $\left(\gamma R T_{w}\right)^{1 / 2}$

$C_{\mu}=$ turbulent viscosity parameter, $v_{T} /\left(L k^{1 / 2}\right)$

$c_{p}=$ specific heat at constant pressure, $\gamma R /(\gamma-$ $1), 1004.5 \mathrm{~J} /(\mathrm{kg} \cdot \mathrm{K})$

$c_{v} \quad=$ specific heat at constant volume, $R /(\gamma-$ 1), $717.5 \mathrm{~J} /(\mathrm{kg} \cdot \mathrm{K})$

$f \quad=\quad$ uniform body force, $\tau_{w} / h$

$h \quad=$ half channel height

$h_{w} \quad=$ wall length scale, $h / R e_{\tau}$

$i=$ turbulence intensity, $(2 k / 3)^{1 / 2} / \bar{U}_{1}$

$k=$ turbulent kinetic energy, $\overline{u_{i} u_{i}} / 2$

$L=$ characteristic length scale of large-scale eddies, $k^{1 / 2} \tau$

$M \quad=$ local Mach number, $\bar{U}_{1} / a$

$M_{g} \quad=$ gradient Mach number, $\Delta_{g} S / a$

$M_{t}^{g} \quad=$ turbulence Mach number, $(2 k)^{1 / 2} / a$

$M_{0} \quad=$ bulk Mach number, $u_{0} / a_{w}$

$m=$ mass fraction of a passive scalar

$P \quad=$ production of $k,-\left|\overline{u_{i} u_{k}}\right| \bar{S}_{k i}$

$P r \quad=$ Prandtl number, 0.7

$P r_{k}=$ turbulence Prandtl number in the $k$ Eq. (15a)

$P r_{k m}=$ molecular Prandtl number in the $k$ Eq. (15a)

$\mathrm{Pr}_{t}=$ turbulence Prandtl number in the mean temperature Eq. (13c)

$p \quad=$ pressure, $\rho R T$

$R=$ gas constant, $c_{p}-c_{v}, 287 \mathrm{~J} /(\mathrm{kg} \cdot \mathrm{K})$

$R e \quad=$ local Reynolds number, $\bar{U}_{1} h / \bar{v}$

$R e_{L}=$ turbulence Reynolds number, $L k^{1 / 2} / \bar{\nu}$

$R e_{\lambda}=$ Taylor-scale Reynolds number, $\left(20 R e_{L} / 3\right)^{1 / 2}$

$R e_{\tau}=$ friction Reynolds number, $\rho_{w} u_{\tau} h / \mu_{w}$

$R e_{0}=$ bulk Reynolds number, $\rho_{0} u_{0} h / \mu_{w}$

$S=$ characteristic strain rate, $\left(2 \bar{S}_{i k}^{d} \bar{S}_{k i}^{d}\right)^{1 / 2}$

$S_{i k} \quad=$ rate-of-strain tensor, $0.5\left(\partial U_{i} / \partial x_{k}+\partial U_{k} / \partial x_{i}\right)$

$S_{i k}^{d}=$ deviatoric part of $S_{i k}, S_{i k}-S_{n n} \delta_{i k} / 3$

$S c \quad=$ Schmidt number, 1.0

$S c_{t}=$ turbulence Schmidt number

$S_{L} \quad=$ standardized source rate in the $L$ equation, see (34)

$S_{\varepsilon} \quad=$ standardized source rate in the $\varepsilon$ equation, see (34)

Received 4 January 2006; revision received 11 May 2006; accepted for publication 22 May 2006. Copyright @ 2006 by the American Institute of Aeronautics and Astronautics, Inc. All rights reserved. Copies of this paper may be made for personal or internal use, on condition that the copier pay the $\$ 10.00$ per-copy fee to the Copyright Clearance Center, Inc., 222 Rosewood Drive, Danvers, MA 01923; include the code $\$ 10.00$ in correspondence with the CCC.

*Professor, Department of Mathematics, 1000 East University Avenue; heinz@uwyo.edu.
$S_{\tau} \quad=\quad$ standardized source rate in the $\tau$ equation, see (34)

$S_{\omega} \quad=$ standardized source rate in the $\omega$ equation, see (25)

$S_{\omega \omega}=$ standardized source rate in the $\omega^{2}$ equation, see (34)

$s=$ standardized characteristic strain rate, $S h_{w} / u_{\tau}$

$T \quad=$ temperature

$T_{w} \quad=$ wall temperature

$U_{i} \quad=$ velocity vector $(i=1,3)$

$u_{i} \quad=$ fluctuating velocity vector, $U_{i}-\bar{U}_{i}(i=1,3)$

$u_{0} \quad=$ bulk velocity, see (5)

$u_{\tau} \quad=$ friction velocity, $\left(\tau_{w} / \rho_{w}\right)^{1 / 2}$

$x_{i} \quad=$ position vector $(i=1,3)$

$\alpha_{1}, \alpha_{2}=$ parameters in the standard model for $S_{w}$, see (16)

$\beta_{\omega} \quad=$ parameter in the $S_{\omega}$ model, see (33)

$\beta_{\omega \omega}=$ parameter in the $S_{\omega \omega}$ model, see (36)

$\gamma=$ ratio of specific heats at constant pressure and constant volume, $c_{p} / c_{v}=1.4$

$\Delta_{g}=$ characteristic length scale of turbulence in the direction of shear, $\kappa\left(h-\left|x_{2}-h\right|\right)$

$\delta_{i j} \quad=$ Kronecker delta

$\varepsilon=$ dissipation rate of $k, \varepsilon_{s}+\varepsilon_{d}, k / \tau$

$\varepsilon_{d} \quad=$ dilatational dissipation rate of $k$

$\varepsilon_{s} \quad=$ solenoidal dissipation rate of $k$

$\kappa \quad=$ von-Karman constant, 0.4

$\mu \quad=$ viscosity, $\mu_{w}\left(T / T_{w}\right)^{0.7}$

$\mu_{T} \quad=$ turbulent viscosity, $C_{\mu}\langle\rho\rangle k \tau$

$\mu_{w} \quad=$ wall viscosity

$v \quad=$ kinematic viscosity, $\mu / \rho$

$v_{T}=$ turbulent kinematic viscosity, $C_{\mu} k \tau$

$\Pi_{d} \quad=$ pressure dilatation, $\overline{(p / \rho)^{\prime \prime} S_{k k}^{\prime \prime}}$

$\rho \quad=$ mass density

$\rho_{w} \quad=\quad$ wall mass density

$\rho_{0} \quad=$ bulk mass density

$\tau=$ dissipation time scale, $1 / \omega$

$\tau_{s}=$ inverse standardized characteristic strain rate $s, 1 / s$

$\tau_{w} \quad=$ wall shear stress

$\omega=$ turbulence frequency, $1 / \tau$

Symbols

$\bar{Q} \quad=$ mass density-weighted mean, $\langle\rho Q\rangle /\langle\rho\rangle$

$\langle Q\rangle \quad=\quad$ ensemble mean of any quantity $Q$

$Q^{\prime \prime} \quad=$ fluctuation of $Q$ around its mass density-weighted mean, $Q-\bar{Q}$

\section{Introduction}

$\mathbf{M}$ OST of the simulations of turbulent reacting flows are performed within the frame of Reynolds-averaged NavierStokes (RANS) methods or more general probability density 
function (PDF) methods (their application allows to describe some important processes exactly, as, for example, chemical conversion processes) [1-9]. The reason for that is given by the fact that the computational costs of RANS and PDF methods are much lower than those of corresponding large eddy simulation (LES) and filter density function (FDF) methods [2,3,6-13]. However, their relative simplicity is also the reason for some significant problems, which limit the accuracy of RANS and PDF methods.

A first problem is given by the fact that there are good concepts available to model the evolution of velocity and scalar fields by stochastic or simpler deterministic methods, but to close such equations one has to provide the turbulence frequency $\omega$ (or dissipation rate $\varepsilon=k \omega$ of the turbulent kinetic energy $k$ ) which determines the characteristic time scale of turbulent motions. Unfortunately, the basis for constructing equations for $\omega$ or $\varepsilon$ is weak because the most important terms in these equations, the standardized source rates $S_{\omega}$ and $S_{\varepsilon}$ [see Eqs. (15b) and (34), respectively], are unknown. To assess these source rates one has to determine the rate at which energy is transferred through the spectrum; this means these source rates are the result of both production of turbulence at large scales and dissipation at small scales [14]. Kolmogorov's notion was that $\omega$ is associated with the smallest scales of turbulence, and thus has no direct interaction with the mean motion $[15,16]$. Correspondingly, he concluded that $S_{\omega}$ should be independent of the production of turbulence and approximated by a constant [15]. Nevertheless, in most of the applications $S_{\omega}$ is considered as a linear function of the productionto-dissipation ratio $P / \varepsilon$ of $k$ [16]. However, the general validity of this assumption is questionable [17,18]. To get further insight into this problem, Yakhot and Orszag [19] and Yakhot et al. [20] addressed this question with regard to the source rate $S_{\varepsilon}$ in the dissipation equation by means of the renormalization group (RNG) theory. Smith and Reynolds [21] found some algebraic errors in the derivation of Yakhot and Orszag [19]. They concluded that this RNG-based theory does not provide support for assuming that $S_{\varepsilon}$ is a function of $P / \varepsilon$. To obtain better agreement with general practice of modeling $S_{\varepsilon}$, Yakhot and Smith [22] revised the RNG theory by modifying several underlying assumptions. This led to the standard form of the dissipation equation plus an additional unknown source term, which was modeled as a function of the dimensionless shear rate of turbulence. However, Smith and Woodruff state, "Even though they may be motivated physically or otherwise, it is evident that many steps of the renormalization-group scale-removal procedure as currently formulated are mathematically not rigorously justified" [23]. Therefore, the question of how the source terms $S_{\omega}$ and $S_{\varepsilon}$ scale with turbulence characteristics cannot be treated as being already clarified.

A second problem is related to the optimization of the performance of turbulence models [2]. The efficiency of turbulence models mainly arises from the fact that turbulence model parameters [as $C_{\mu}$, see relation (14)] are introduced via the parametrization of correlations of turbulent velocities and scalars which appear as unknowns in turbulence models. Originally, such model parameters were assumed to be constant [24], but many investigations indicated significant shortcomings as a consequence of this assumption. This concerns, in particular, the modeling of wall-bounded flows, which has to be considered in most of the applications. It turned out that the performance of turbulence models for such flows can be significantly improved by adopting varying turbulence model parameters, so that the damping effect of walls can be taken into account. Basically, two concepts were applied previously for the construction of such damping functions $[3,16]$ : the scaling of coefficients in terms of normalized wall distances, or their scaling with a turbulence Reynolds number. However, the application of wall distance concepts becomes problematic in complex geometries (flow along a right angled corner), and the generality of such parameterizations is questionable (see the detailed discussion in Sec. II.B) [3]. Regarding the use of $R e_{L}$, Rodi and Mansour observed that such parameterizations are not supported by direct numerical simulation (DNS) [25]. Thus, the question of how variations of turbulence model parameters should be described cannot be seen to be already solved. Consequently, a general concept for the optimization of the performance of turbulence models is unavailable at present.

A third problem concerns the development of solutions for the two problems described in the preceding paragraphs (or, more general, the development of turbulence models) for variable-density flows, which is relevant to turbulent combustion calculations [26-42]. Compressibility effects that were observed in such flows may be differentiated, basically, into dilatational and structural compressibility effects [7,27]. Dilatational compressibility effects were observed in homogeneous shear flows [26,42]; independent of the gradient Mach number [see relation (12)] one finds that the ratio of both the dilatational dissipation rate and pressure-dilatation correlation to the solenoidal dissipation rate is about $10 \%$ [37]. However, the relevance of dilatational compressibility effects to wall-bounded flows seems to be very low [29-36]. In contrast to that, structural compressibility effects (changes of the dimensionless anisotropy tensor due to a reduction of the turbulent kinetic energy redistribution) were found to have a very significant effect on the production and dissipation of turbulence in homogeneous shear flows [42], which requires corresponding modifications of turbulence models [37]. With regard to wall-bounded flows there is certainly the need for further investigations of their significance and of appropriate ways to incorporate these effects in turbulence models. It has to be clarified, for example, whether the aforementioned parameters $S_{\omega}$ and $C_{\mu}$ are significantly affected by such structural changes, and whether the scaling of structural compressibility effects in terms of the gradient Mach number is an appropriate concept also for wall-bounded flows.

The three problems pointed out in the preceding paragraphs will be addressed here. By analyzing recently obtained DNS data of supersonic channel flow at different Reynolds and Mach numbers [29-32], these investigations will be performed in the following way. The DNS data applied will be described in Sec. II. Questions addressed in this way concern the scaling of wall effects, Reynolds and Mach number variations, and the mechanism of production of turbulent kinetic energy. In Sec. III, these DNS data will be used to calculate the parameters of various turbulence models to find answers to the aforementioned questions (the mechanism of the turbulence frequency production, the scaling of the damping effect of walls, and compressibility effects). Section IV deals with conclusions of this study.

\section{Direct Numerical Simulation}

Compressible flow of air through a channel of infinitely large plates (separated by a distance $2 h$ ) is considered; see the illustration in Fig. 1. The flow is driven by a uniform body force. No-slip and impermeability conditions are applied to the velocity field at the walls, and periodic boundary conditions are used in streamand spanwise directions. Both channel walls are cooled and kept at constant temperature so that there is heat transfer out of the channel allowing supersonic fully-developed flow. A passive scalar is injected at the lower wall and removed at the upper wall [29-32].

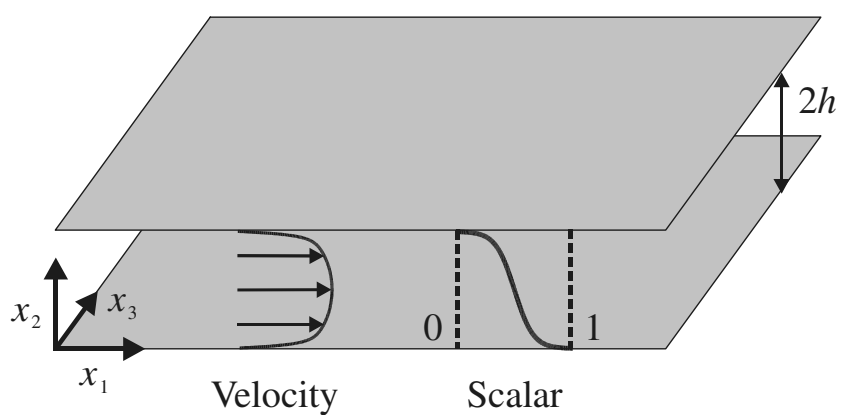

Fig. 1 A sketch of the flow considered. Mean velocity and scalar profiles appear only in wall-normal direction $x_{2}$. 
Table 1 Characteristic simulation data for the IL, CL, and CH cases defined in Table 2. $N_{1}, N_{2}$, and $N_{3}$ denote the number of grid points in the $x_{1}-, x_{2}$, and $x_{3}$-directions, respectively. $L_{1}, L_{2}$, and $L_{3}$ are the corresponding domain lengths. $\Delta x_{1}^{+}, \Delta x_{2}^{+}$, and $\Delta x_{3}^{+}$refer to the node distance normalized on the viscous length scale $h_{w}=v_{w} / u_{\tau}$ (regarding $\Delta x_{2}^{+}$the minimal and maximal values are given).

\begin{tabular}{lccccccccrr}
\hline \hline Case & $N_{1}$ & $N_{2}$ & $N_{3}$ & $L_{1} / h$ & $L_{2} / h$ & $L_{3} / h$ & $\Delta x_{1}^{+}$ & $\Delta x_{2 \min }^{+}$ & $\Delta x_{2 \max }^{+}$ & $\Delta x_{3}^{+}$ \\
\hline IL & 192 & 129 & 160 & 9.6 & 2 & 6 & 9.12 & 1.02 & 4.21 & 6.84 \\
CL & 512 & 221 & 256 & $4 \pi$ & 2 & $4 \pi / 3$ & 13.65 & 0.89 & 9.38 & 8.91 \\
CH & 512 & 301 & 256 & $6 \pi$ & 2 & $4 \pi / 3$ & 37.89 & 1.27 & 13.35 & 16.85 \\
\hline \hline
\end{tabular}

\section{A. The Numerical Method}

The flow dynamics are described by the compressible NavierStokes equations for the mass density, velocity, temperature, and mass fraction of a passive scalar:

$$
\begin{gathered}
\frac{\partial \rho}{\partial t}+U_{k} \frac{\partial \rho}{\partial x_{k}}=-\rho S_{k k} \\
\frac{\partial U_{i}}{\partial t}+U_{k} \frac{\partial U_{i}}{\partial x_{k}}=\frac{2}{\rho} \frac{\partial}{\partial x_{k}} \mu S_{i k}^{d}-\frac{1}{\rho} \frac{\partial p}{\partial x_{i}}+\frac{1}{\rho}\left(f+\frac{\partial\langle p\rangle}{\partial x_{1}}\right) \delta_{i 1} \\
\frac{\partial T}{\partial t}+U_{k} \frac{\partial T}{\partial x_{k}}=\frac{1}{\rho} \frac{\partial}{\partial x_{k}} \frac{\gamma \mu}{\operatorname{Pr}} \frac{\partial T}{\partial x_{k}}-(\gamma-1) S_{k k} T+\frac{2}{\rho c_{v}} \mu S_{i j}^{d} S_{j i}^{d} \\
\frac{\partial m}{\partial t}+U_{k} \frac{\partial m}{\partial x_{k}}=\frac{1}{\rho} \frac{\partial}{\partial x_{k}} \frac{\mu}{\operatorname{Sc}} \frac{\partial m}{\partial x_{k}}
\end{gathered}
$$

Here, $\delta_{i j}$ is the Kronecker delta and the sum convention is applied throughout this paper. The pressure is given by the thermal equation of state $p=\rho R T$. In (1b), a force $f$ [which is determined by relation (19)] is introduced on the right-hand side which replaces the ensemble-averaged pressure gradient $\partial\langle p\rangle / \partial x_{1}$. The rate-of-strain tensor and its deviatoric part are defined by

$$
S_{i k}=\frac{1}{2}\left(\frac{\partial U_{i}}{\partial x_{k}}+\frac{\partial U_{k}}{\partial x_{i}}\right), \quad S_{i k}^{d}=\frac{1}{2}\left(\frac{\partial U_{i}}{\partial x_{k}}+\frac{\partial U_{k}}{\partial x_{i}}-\frac{2}{3} \frac{\partial U_{n}}{\partial x_{n}} \delta_{i k}\right)
$$

The closure of $(1 \mathrm{a}-1 \mathrm{~d})$ requires the definition of molecular properties. The expression

$$
\mu=\mu_{w}\left(\frac{T}{T_{w}}\right)^{0.7}
$$

is used for the dynamic viscosity.

The solution of $(1 \mathrm{a}-1 \mathrm{~d})$ was performed by adopting these equations in a pressure-velocity-entropy formulation [43]. The compact fifth-order upwind scheme of Adams and Shariff [44] was used to discretize the hyperbolic (Eulerian) terms. The viscous and heat conduction terms were discretized with the compact sixth-order scheme of Lele [45]. The solution was advanced in time with a thirdorder "low-storage" Runge-Kutta scheme proposed by Williamson [46]. Equidistant grids were used in $\left(x_{1}, x_{3}\right)$-directions. In the wallnormal $x_{2}$-direction, points were clustered following tanh-functions [39]. Guideline for the domain size and resolution described in Table 1 was given by the simulations of Coleman et al. [28], Kim et al. [47] and Moser et al. [48]. The suitability of the domain size and resolution applied was shown by a detailed analysis of onedimensional power density spectra and two-point correlations of flow variables [32]. Regarding the Mach numbers applied, there was no need to resolve shock waves (shocklets) which were not observed. The numerical algorithm has been previously validated by Lechner et al. [39] whose results for a bulk Mach number $M_{0}=1.5$ agreed well with the results of Coleman et al. [28]. As shown in Foysi et al., [31,32] the bulk Mach number $M_{0}=0.3$ simulation (the incompressible and low-Reynolds number (IL) case: see Tables 1 and 2) agreed well with corresponding DNS data of Moser et al. [48] (which did not involve passive scalar transport).
Table 2 The friction Reynolds number, bulk Reynolds number, and Mach number for the cases IL, $\mathrm{CL}$, and $\mathrm{CH}$ considered. In addition, the scaling parameters $\mu_{w}, u_{\tau}$, and $h_{w}$ are given in their corresponding units $(\mathrm{kg} /(\mathrm{m} \cdot \mathrm{s}), \mathrm{m} / \mathrm{s}, \mathrm{m}$, respectively).

\begin{tabular}{lrrrccc}
\hline \hline Case & \multicolumn{1}{c}{$R e_{\tau}$} & \multicolumn{1}{c}{$R e_{0}$} & $M_{0}$ & $\mu_{w}$ & \multicolumn{1}{c}{$u_{\tau}$} & $h_{w}$ \\
\hline IL & 181 & 2820 & 0.3 & 0.05 & 7.55 & $5.49 \times 10^{-3}$ \\
CL & 556 & 6000 & 3.0 & $1.91 \times 10^{-5}$ & 50.4 & $8.99 \times 10^{-6}$ \\
CH & 1030 & 11310 & 3.5 & $1.91 \times 10^{-5}$ & 48.9 & $4.86 \times 10^{-6}$ \\
\hline \hline
\end{tabular}

The stationary ensemble (Reynolds) and mass density-weighted (Favre) means considered were obtained by averaging over the homogeneous stream- and spanwise directions (such that all averages obtained only depend on the wall-normal coordinate $x_{2}$ ). Additional averaging over values of flow variables at the same wall distance was performed by adopting the channel symmetry in wallnormal $x_{2}$-direction. After a time $h / u_{\tau}$, the distance covered by the fluid is approximately equal to the half channel height. Stationary states of flow variables were obtained after $25 h / u_{\tau}$ [compressible and low-Reynolds number (CL) case] and $50 h / u_{\tau}$ [IL and compressible and high-Reynolds number $(\mathrm{CH})$ cases]. Then, averaging in time was performed by adopting about $10^{3}$ instantaneous fields. Thus, flow statistics were obtained on the basis of more than $3 \times 10^{7}$ samples values of flow variables.

Equations (1a-1d) were solved for three sets of the friction Reynolds number, bulk Reynolds number, and Mach number. These parameters are defined by

$$
R e_{\tau}=\frac{\rho_{w} u_{\tau} h}{\mu_{w}}, \quad R e_{0}=\frac{\rho_{0} u_{0} h}{\mu_{w}}, \quad M_{0}=\frac{u_{0}}{a_{w}}
$$

They may be seen as dimensionless measures for the friction velocity, bulk velocity, and inverse of sound velocity at the wall. These velocities are given by

$$
u_{\tau}=\sqrt{\frac{\tau_{w}}{\rho_{w}}}, \quad u_{0}=\frac{1}{h} \int_{0}^{h} \mathrm{~d} x_{2} \bar{U}_{1}, \quad a_{w}=\sqrt{\gamma R T_{w}}
$$

$\rho_{0}$ is defined in correspondence to $u_{0}$. The values for $R e_{\tau}, R e_{0}$, and $M_{0}$ considered in this way are presented in Table 2. This table shows that the cases IL, CL, and $\mathrm{CH}$ differ by growing Reynolds and Mach numbers $R e_{\tau}, R e_{0}$, and $M_{0}$.

\section{B. The Scaling of Flow Characteristics}

Let us consider the scaling of wall effects to prepare the discussion of characteristic flow features in dependence on Reynolds and Mach numbers. One may consider two constraints for such a scaling. First of all, it should be correct; this means it should be in consistency with basic physical principles and supported by DNS or experimental data. Second, the scaling should have a relatively broad range of applicability. As shown by Rodi and Mansour [25], previously applied scalings in terms of turbulence Reynolds numbers do not satisfy the first constraint because support by DNS data is unavailable. Scaling concepts based on wall distances do not provide turbulence models that satisfy the first constraint because they are not invariant under the Galilean transformation (such models can be seen to be physically incorrect according to Yakhot et al. [20]). Scaling concepts based on wall distances also do not satisfy the second constraint: one needs different (inner and outer) scalings for different 
flow regions $[3,16]$, and inner scaling turned out to be inapplicable to compressible flow (the use of the semilocal scaling of Huang et al. [38] gives better performance, but the improvement is only partial) [31]. It is worth noting that there is no reason to expect that models developed for channel flow are applicable to flow along a right angled corner (where the walls produce a complicated superposition of damping effects) or more complex geometries. In correspondence to compressibility effects, mass density changes due to chemical reactions may well have a relevant influence on wall damping effects, which cannot be considered on the basis of such scaling concepts. There is, therefore, an obvious need for the development of wall effect scalings that are independent of wall parameters [49].

A valuable guideline for the choice of an appropriate scaling parameter can be obtained by having a closer look at the balance of mean forces for the flows considered. As shown in detail in Sec. III [see relation (18)], the uniform body force realizes the following shear stress balance,

$$
\left(\langle\mu\rangle+\mu_{T}\right)\left|\frac{\partial \bar{U}_{1}}{\partial x_{2}}\right|=f\left|h-x_{2}\right|
$$

where a flux-gradient relation is used for the turbulent momentum flux. For the flow considered we may generalize $\left|\partial \bar{U}_{1} / \partial x_{2}\right|$ by the characteristic mean flow frequency scale $S=\left(2 \bar{S}_{i k}^{d} \bar{S}_{k i}^{d}\right)^{1 / 2}$. By adopting $f=\tau_{w} / h$ derived in Sec. III and introducing the parameter $s=S \mu_{w} / \tau_{w}$, relation (6) may be written

$$
\frac{\mu_{w}}{\langle\mu\rangle+\mu_{T}}=\frac{s}{\left|1-x_{2} / h\right|}
$$

A plot of $s /\left|1-x_{2} / h\right|$ against $s$ is given in Fig. 2a. This figure shows that the three cases considered may be well approximated by the following linear function of $s$

$$
\frac{s}{\left|1-x_{2} / h\right|}=0.02+0.98 s
$$

Relations (7) and (8) indicate the suitability of $s$ as scaling parameter. In contrast to scalings based on wall distances, one finds that a relevant property of turbulence (the normalized inverse effective viscosity) is described consistently for all the flow independent of Reynolds and Mach numbers by a linear relation. In particular, Fig. 2a shows that the ratio $s /\left|1-x_{2} / h\right|$ is hardly affected by $\left|1-x_{2} / h\right|$. The reason for that can be seen in Fig. $2 \mathrm{~b}$; the variation of $\left|1-x_{2} / h\right|$ within the range of significant $s$ variations is relatively small $\left(0.82 \leq\left|1-x_{2} / h\right| \leq 1\right.$ for $\left.0.1<s<1\right)$. Thus, the inverse effective viscosity is linearly correlated to $s$, which means that $s$ is the appropriate parameter for the scaling of variations of this turbulence property.

Regarding the properties of $s$ it is relevant to note that $s$ is bounded by zero and unity $(0 \leq s \leq 1)$; $s$ cannot become negative such that its value is zero at the centerline, and its maximum one is found at the wall (this maximum of $s$ has to be equal to one because both $\left|1-x_{2} / h\right|$ and $\left(\langle\mu\rangle+\mu_{T}\right) / \mu_{w}$ are equal to one at the wall). To use the scaling of $s$ also for the scaling of other flow variables considered next, it is convenient to write $s$ in terms of a velocity scale $u_{\tau}$ and a

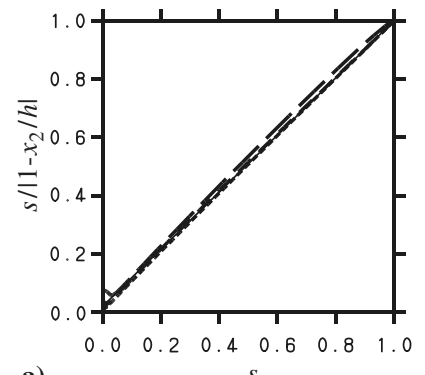

a)

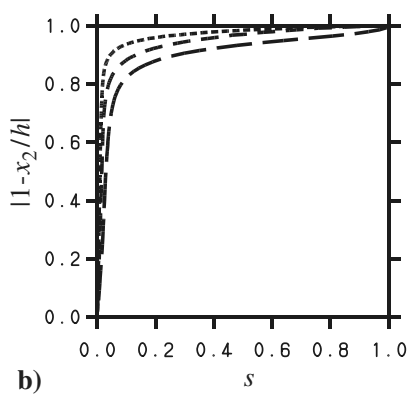

Fig. 2 DNS data for $s /\left|1-x_{2} / h\right|$ and $\left|1-x_{2} / h\right|$ are shown against $s$ (IL: long dashes; CL: medium dashes; $\mathrm{CH}$ : short dashes). The linear function $0.02+0.98 s$ is given in a) for a comparison (solid line) length scale $h_{w}$. By adopting relation (5) between $\tau_{w}$ and $u_{\tau}, s$ may be written

$$
s=\frac{h_{w}}{u_{\tau}} S
$$

Here, we introduced the wall length scale, which is defined by

$$
h_{w}=\frac{u_{\tau} \mu_{w}}{\tau_{w}}=\frac{h}{R e_{\tau}}
$$

The values of $u_{\tau}, h_{w}$, and $\mu_{w}$ for the three cases considered may be found in Table 2. The normalization of $s$ corresponds to the use of inner scaling parameters $u_{\tau}$ and $h_{w}$ (which is plausible because relevant changes of $s$ are found within the inner wall region; see Fig. 2b).

What are the differences between a scaling based on wall distances and a scaling in terms of $s$ ? The parameter $s$ characterizes mean flow changes which determine essentially the production of turbulence ( $s$ characterizes the magnitude of the deviatoric rate-of-strain tensor which controls the production of turbulence and Reynolds stresses in algebraic turbulence models). The idea of using $s$ for the scaling of flow properties is to explain changes of turbulence properties in relation to mean flow changes which produce turbulence. Does a scaling in terms of $s$ satisfy the aforementioned constraints for scaling concepts? The use of $s$ satisfies the first constraint: such a scaling is consistent with basic physical principles ( $s$ is an invariant of the rate-of-strain tensor such that its value is independent of the coordinate system applied) and supported by DNS (Fig. 2a illustrates the suitability of $s$ as scaling parameter). The second constraint is also satisfied. Figure 2a shows that this scaling applies to all the flow and all Reynolds and Mach numbers considered. The reference to a local flow state which is independent of wall properties is advantageous for simulations of flows through complex geometries, this means under conditions where a complicated superposition of damping effects is found. There is no need to specify the reasons for variations of $s$ which enables applications to many other conditions than considered here (applications to multicomponent reacting flow where variations of $s$ may be produced, for example, by chemical reactions). A scaling in terms of $s$ offers, therefore, significant advantages compared with scaling concepts that take the wall distance explicitly into account.

As shown in Fig. 3, the use of $s$ as scaling parameter is very helpful for the explanation of flow structures. Figures $3 a$ and $3 b$ show that this scaling linearizes the profiles of the normalized mean temperature $\bar{T} / T_{w}$ and inverse mass density $\rho_{w} /\langle\rho\rangle$. There is hardly any observable difference between the corresponding curves such that $\bar{T} / T_{w} \approx \rho_{w} /\langle\rho\rangle$. Hence, $\langle p\rangle=\langle\rho\rangle R \bar{T} \approx \rho_{w} R T_{w}=p_{w}$, which means that the mean pressure is approximately equal to its wall value $p_{w}$. Figure $3 \mathrm{c}$ shows that $\bar{m}$ is, basically, also linearly correlated to $s$. Another advantage of using $s$ as scaling parameter may be seen in Fig. 3d: the scaling applied enables a good spatial resolution of the sharp peaks of the turbulent kinetic energy near the walls. This figure reveals a significant compressibility effect. Instead of the relatively uniform $k^{1 / 2} / u_{\tau}$ distribution found for incompressible flow within the range $0.1<s<0.8$, we see that compressibility increases significantly the peak value of $k^{1 / 2} / u_{\tau}$, and it causes an approximately linear decay of $k^{1 / 2} / u_{\tau}$ towards zero at the wall. This behavior of the energy of turbulent fluctuations shows similarities to the behavior of mean temperatures that indicate the energy of thermal fluctuations. It is a plausible finding that an increase of mean mass density with decreasing wall distance in compressible flows implies a decrease of turbulent kinetic energy. The normalized dissipation rate $\varepsilon h_{w} / u_{\tau}^{3}$ of $k$ is shown in Fig. $3 \mathrm{e}$ ( $\varepsilon$ is often considered as the sum of solenoidal and dilatational contributions, $\varepsilon=\varepsilon_{s}+\varepsilon_{d}$, but there is no need for this differentiation here because dilatational contributions are extremely small: one finds $\left.\varepsilon_{d} / \varepsilon_{s}<0.001\right)$. We see that the distribution of $\varepsilon h_{w} / u_{\tau}^{3}$ has similarities to the $k^{1 / 2} / u_{\tau}$ distribution (there is again a compressibility effect; the increase of mean mass density with decreasing wall distance in compressible flows implies a higher 


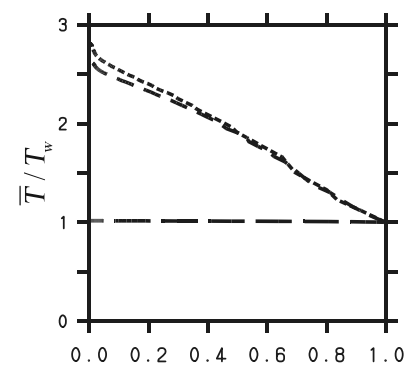

a)
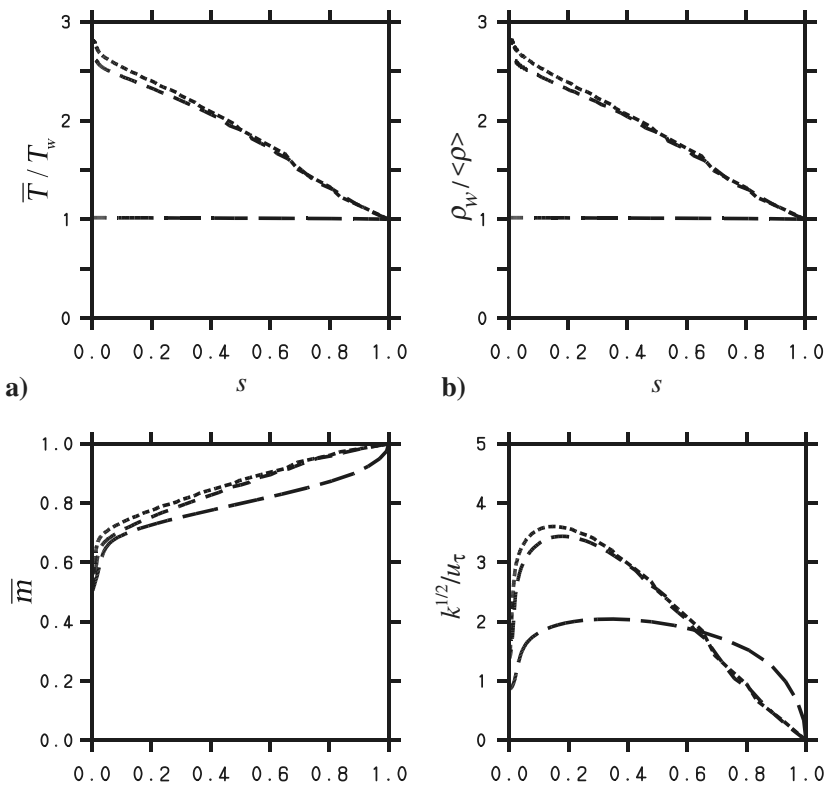

c)

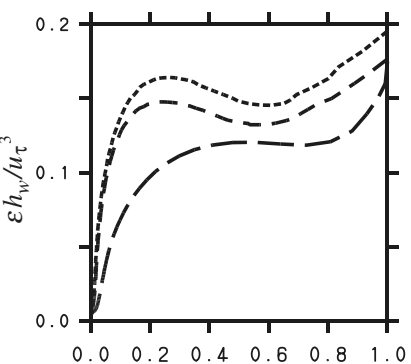

e)

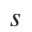

d)

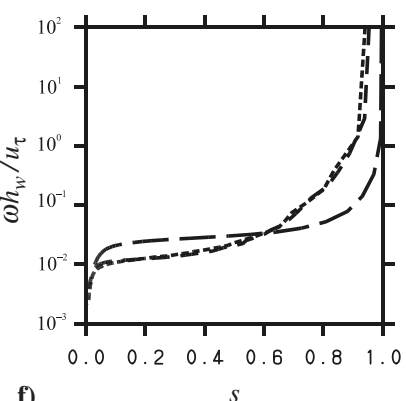

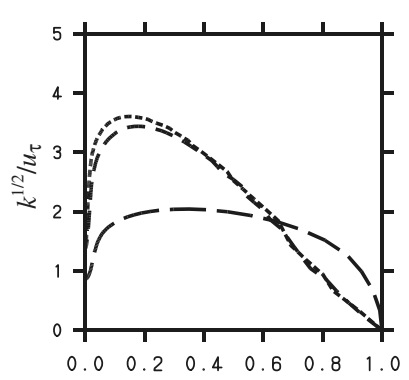

Fig. 3 Variations of mean flow and turbulence properties with $s$. The same line coding is used as in Fig. 2.

dissipation rate of turbulent kinetic energy) with one exception: $\varepsilon h_{w} / u_{\tau}^{3}$ tends to level off after the maximum of $k^{1 / 2} / u_{\tau}$ is reached. The normalized characteristic frequency of turbulence $\omega h_{w} / u_{\tau}$ is shown in Fig. 3f. As may be seen, $\omega h_{w} / u_{\tau}$ varies over many orders of magnitude. The huge variations of $\omega h_{w} / u_{\tau}$ also indicate that the use of derivatives of $\omega h_{w} / u_{\tau}$ may become problematic for $0.8<s$. Additional illustrations of the accuracy of these DNS data will be given next in conjunction with the calculation of nondimensional flow characteristics (which actually represent rewritten balance equations for the corresponding variables). Further discussions of the accuracy of these DNS data may be found elsewhere [31,32].

\section{Reynolds and Mach numbers}

As may be seen in Table 2, the cases IL, CL, and $\mathrm{CH}$ differ by growing Reynolds and Mach numbers $R e_{\tau}, R e_{0}$, and $M_{0}$. However, these global parameters do not reflect local flow characteristics. This may be seen by considering the local Reynolds number and Mach number, which are defined in the following way:

$$
\operatorname{Re}=\frac{\bar{U}_{1} h}{\bar{v}}, \quad M=\frac{\bar{U}_{1}}{a}
$$

Here, $\bar{U}_{1}$ is the mean streamwise velocity and $\bar{v}=\langle\mu\rangle /\langle\rho\rangle$ is the mean kinematic viscosity. The corresponding curves of $R e$ and $M$ are given in Figs. 4a and 4b. One observes that the local Reynolds numbers of IL and CL and the local Mach numbers of CL and CH are very similar. Thus, the comparison of IL and CL shows the Mach number effect whereas the Reynolds number effect follows from comparing $\mathrm{CL}$ and $\mathrm{CH}$.

The turbulence is characterized by the turbulence Reynolds number, Taylor-scale Reynolds number, turbulence Mach number,
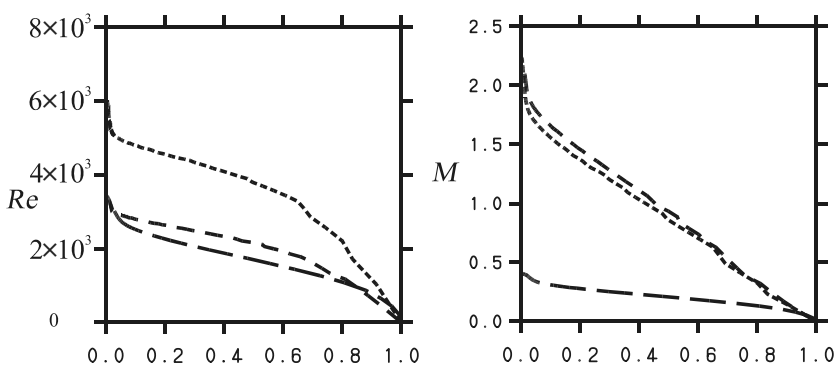

a)

b)
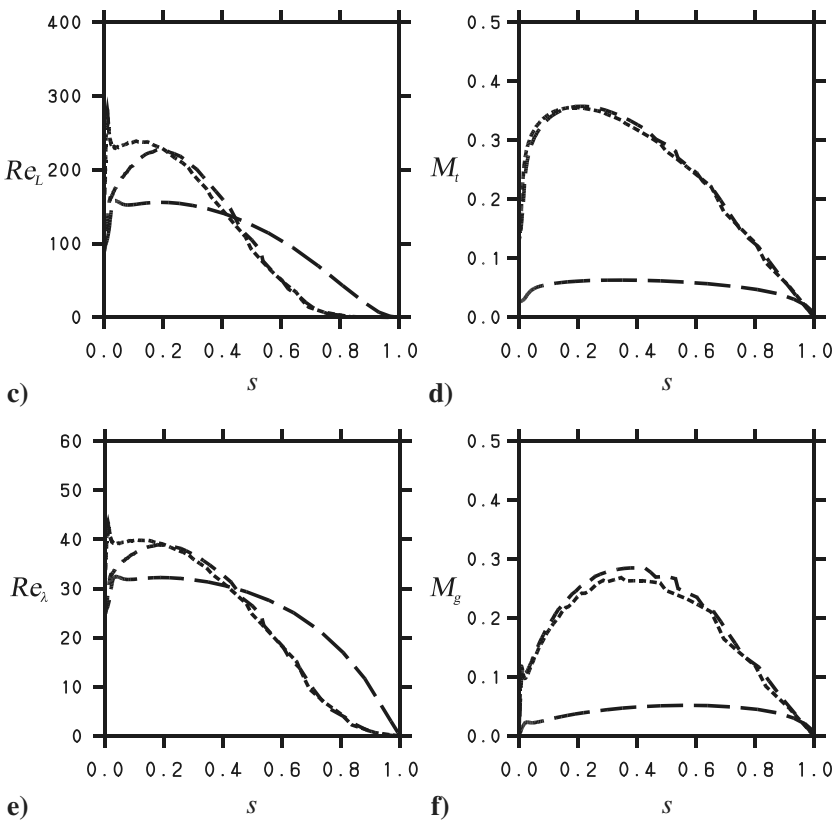

Fig. 4 Variations of Reynolds and Mach numbers with $s$. The same line coding is used as in Fig. 2.

and gradient Mach number, which are defined by

$$
\begin{aligned}
R e_{L}=\frac{L \sqrt{k}}{\bar{v}}, \quad R e_{\lambda} & =\sqrt{\frac{20}{3} R e_{L}}, \quad M_{t}=\frac{\sqrt{2 k}}{a} \\
M_{g} & =\frac{\Delta_{g} S}{a}
\end{aligned}
$$

Here, $L=k^{1 / 2} \tau=k^{3 / 2} / \varepsilon$ denotes a characteristic length scale of large-scale eddies. The corresponding curves for $R e_{L}, R e_{\lambda}, M_{t}$, and $M_{g}$ are given in Figs. 4c-4f. $R e_{L}$ reveals similarities to the distribution of $k^{1 / 2} / u_{\tau}$; see the preceding discussion. A Taylor-scale Reynolds number $R e_{\lambda} \approx 40$ was found in investigations of homogeneous shear flows [42]. We see that the Taylor-scale Reynolds numbers here are approximately in the same range for $s<0.4$. In contrast to that, the turbulence Mach number and gradient Mach number are found to be small compared with values observed in homogeneous shear flows [42].

\section{Basic Flow Characteristics}

Basic flow characteristics are given in Fig. 5. The normalized production $P /(S k)=\left|\overline{u_{1} u_{2}}\right| / k$ and inverse dissipation $S k / \varepsilon$ of turbulent kinetic energy are shown in Figs. 5a and $5 \mathrm{~b}$. In homogeneous shear flows, both the quantities $P /(S k)$ and $S k / \varepsilon$ were found to be primarily affected by structural compressibility effects: they scale with the gradient Mach number [37]. For the wall-bounded flows considered here, the influence of such compressibility effects is also found to be significant. In particular, one finds the same trends as given with regard to homogeneous shear flows: both the normalized production and dissipation decrease with growing compressibility. Reynolds number effects are found to be small. In contrast to findings 


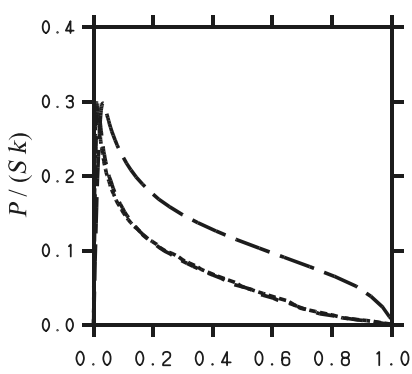

a)
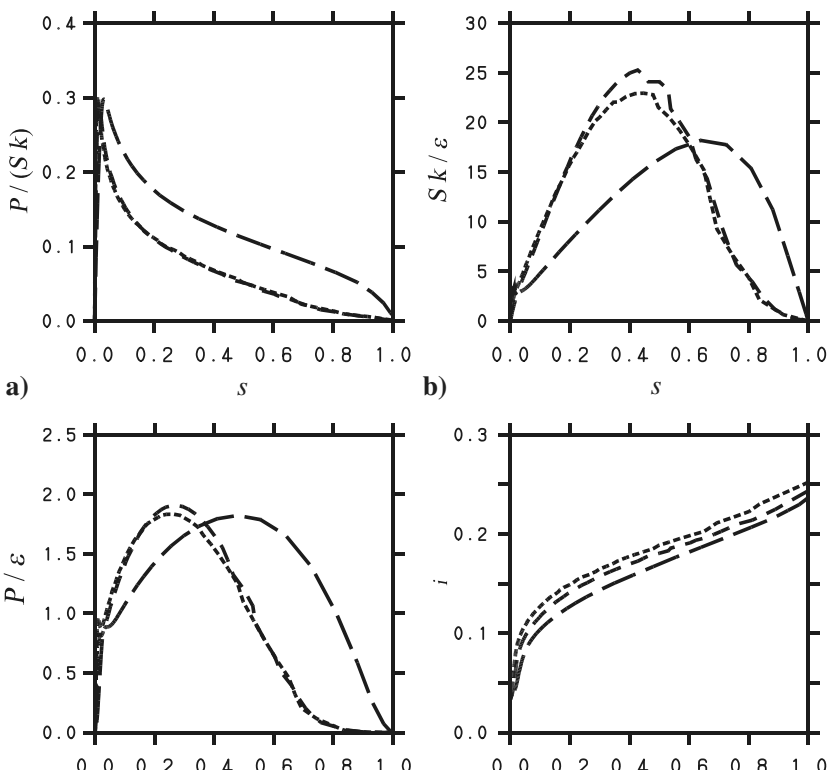

c)

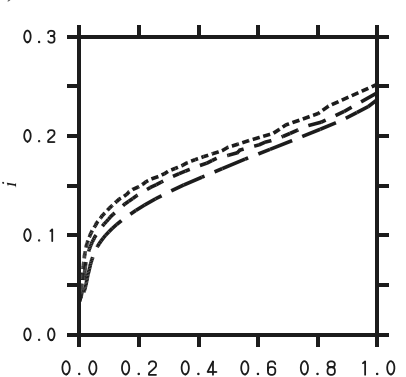

d)
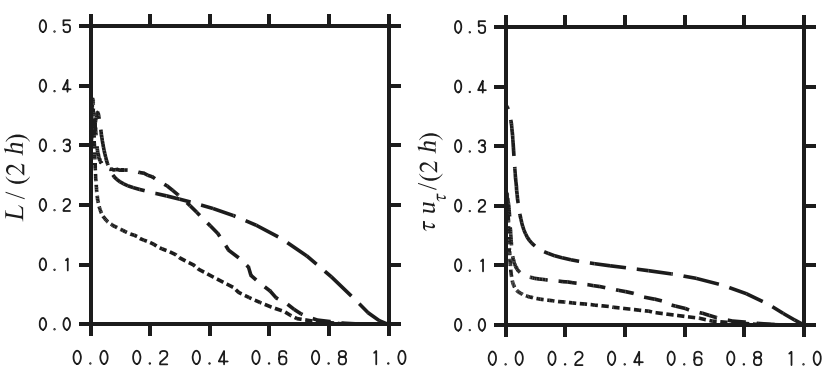

f)

fields, and models for the evolution of characteristic time scales of turbulent motions. The calculation of characteristic time scales is, basically, the same in all types of turbulence models, but there is a variety of ways to construct velocity and scalar models (one can use PDF methods or their implied Reynolds stress models, or one can use two-equation turbulence models) $[3,6,7,16]$. PDF and corresponding Reynolds stress models are relatively well developed; for the flow considered one can apply, for example, the Langevin model or corresponding Reynolds stress models [37,50]. Two-equation turbulence models are the models that are applied (and will be applied for the foreseeable future) to the majority of all practical calculations $[2,3,6,7,16,51]$, but their use is faced with a variety of problems. To address these questions discussed in the introduction, we will consider, therefore, this type of turbulence model.

\section{A. The $k-\omega$ Turbulence Model}

The questions considered will be addressed first on the basis of a $k-\omega$ turbulence model: compared with a $k-\varepsilon$ model, this model has the advantage that it can be applied well into the viscous sublayer, whereas the $k-\varepsilon$ model (with wall functions) requires the first grid point away from the wall to lie in the log layer $[6,16]$. It is, however, worth noting that the following discussions will not be limited to the consideration of the $k-\omega$ turbulence model, but the suitability of other two-equation turbulence models will be also addressed (see Sec. III.D). By adopting algebraic approximations for turbulent fluxes $[3,5-7,16]$, the transport equations for the ensemble-averaged mass density $\langle\rho\rangle$, mass density-weighted velocities $\bar{U}_{i}$, temperature $\bar{T}$, and mass fraction $\bar{m}$ read

$$
\begin{gathered}
\frac{\partial\langle\rho\rangle}{\partial t}+\bar{U}_{k} \frac{\partial\langle\rho\rangle}{\partial x_{k}}=-\langle\rho\rangle \bar{S}_{k k} \\
\frac{\partial \bar{U}_{i}}{\partial t}+\bar{U}_{k} \frac{\partial \bar{U}_{i}}{\partial x_{k}}=\frac{2}{\langle\rho\rangle} \frac{\partial}{\partial x_{k}}\left(\langle\mu\rangle+\mu_{T}\right) \bar{S}_{i k}^{d}-\frac{1}{\langle\rho\rangle} \frac{\partial(\langle p\rangle+2\langle\rho\rangle k / 3)}{\partial x_{i}} \\
+\frac{1}{\langle\rho\rangle}\left(f+\frac{\partial\langle p\rangle}{\partial x_{1}}\right) \delta_{i 1} \\
\frac{\partial \bar{T}}{\partial t}+\bar{U}_{k} \frac{\partial \bar{T}}{\partial x_{k}}=\frac{1}{\langle\rho\rangle} \frac{\partial}{\partial x_{k}} \gamma\left(\frac{\langle\mu\rangle}{P r}+\frac{\mu_{T}}{P r_{t}}\right) \frac{\partial \bar{T}}{\partial x_{k}}-(\gamma-1) \bar{S}_{k k} \bar{T} \\
+\frac{\varepsilon}{c_{v}}+\frac{\bar{v}}{c_{v}} S^{2} \\
\frac{\partial \bar{m}}{\partial t}+\bar{U}_{k} \frac{\partial \bar{m}}{\partial x_{k}}=\frac{1}{\langle\rho\rangle} \frac{\partial}{\partial x_{k}}\left(\frac{\langle\mu\rangle}{S c}+\frac{\mu_{T}}{S c_{t}}\right) \frac{\partial \bar{m}}{\partial x_{k}}
\end{gathered}
$$

The mean pressure is given by $\langle p\rangle=\langle\rho\rangle R \bar{T}$. For the turbulent viscosity, we apply the parameterization

$$
\mu_{T}=C_{\mu}\langle\rho\rangle \frac{k}{\omega}
$$

Here, $C_{\mu}$ is a parameter that has to be calculated and $\omega=1 / \tau=\varepsilon / k$ refers to the turbulence frequency. It is assumed that the turbulent kinetic energy $k$ and $\omega$ obey the equations

$$
\frac{\partial k}{\partial t}+\bar{U}_{k} \frac{\partial k}{\partial x_{k}}=\frac{1}{\langle\rho\rangle} \frac{\partial}{\partial x_{k}}\left(\frac{\langle\mu\rangle}{P r_{k m}}+\frac{\mu_{T}}{P r_{k}}\right) \frac{\partial k}{\partial x_{k}}+C_{\mu} S^{2} \frac{k}{\omega}-k \omega
$$
increases both $L /(2 h)$ and $\tau u_{\tau} /(2 h)$ due to the reduction of turbulent friction. As it has to be expected, a lower level of compressibility also increases the characteristic length and time scales of turbulent eddies.

\section{A $k-\omega$ Analysis of DNS Data}

The DNS data described in Sec. II will be considered now within the frame of a turbulence model, i.e., the turbulence model parameters will be calculated such that the model predictions agree with the corresponding DNS data. Turbulence models involve two basic ingredients: models for the evolution of velocity and scalar

$$
\frac{\partial \omega}{\partial t}+\bar{U}_{k} \frac{\partial \omega}{\partial x_{k}}=\frac{1}{\langle\rho\rangle} \frac{\partial}{\partial x_{k}}\left(\frac{\langle\mu\rangle}{P r_{k m}}+\frac{\mu_{T}}{P r_{k}}\right) \frac{\partial \omega}{\partial x_{k}}-S_{\omega} \omega^{2}
$$

Equation (15a) involves a molecular Prandtl number in analogy to $\mathrm{Pr}$ and $S c$ in (13c) and (13d). Regarding Eq. (15b) we assumed that this equation may be obtained by rewriting the turbulent kinetic energy equation (by adopting $k=\varepsilon / \omega$ ). Then, the effective viscosity in the turbulence frequency equation is the same as in the turbulent kinetic 
energy equation, as it is usually assumed [16]. $S_{\omega}$ refers to the source rate in the turbulence frequency equation, which is usually parameterized by the expression

$$
S_{\omega}=\alpha_{2}-\alpha_{1} \frac{C_{\mu}}{\omega^{2}} S^{2}
$$

A discussion of the plausibility of the parameterization (16) may be found in the textbooks of Wilcox ([16], p. 120) and Pope ([3], p. 377). Applications of this model are described by Wilcox [16]. The parameters $\alpha_{1}$ and $\alpha_{2}$ have to be calculated. $C_{\mu} S^{2} / \omega^{2}$ represents the modeled production-to-dissipation ratio of turbulent kinetic energy [see (15a)]. Regarding the structure of (15a) and (15b) it is worth noting that the mean dilatation is negligible for the flow considered. In correspondence to the negligible influence of dilatational dissipation effects one finds that the contribution of the pressure dilatation is extremely small: we have $\left|\Pi_{d} /(\langle\rho\rangle \varepsilon)\right|<0.008$.

\section{B. The Model for the Case Considered}

To prepare the discussion of the model parameters implied by DNS data in Sec. III.C, we specify the turbulence model (13a-13d), (15a), and (15b) for the compressible channel flow considered. The $\partial\langle p\rangle / \partial x_{1}$ terms in (13b) cancel each other and the remaining quantities vary only in the $x_{2}$-direction. The left-hand sides vanish due to the consideration of the stationary state and $\bar{U}_{k}=\bar{U}_{1} \delta_{k 1}$. Hence, the momentum Eq. (13b) may be written

$$
0=\left[\frac{\partial}{\partial x_{2}}\left(\langle\mu\rangle+\mu_{T}\right) \frac{\partial \bar{U}_{1}}{\partial x_{2}}+f\right] \delta_{i 1}-\frac{\partial(\langle p\rangle+2\langle\rho\rangle k / 3)}{\partial x_{2}} \delta_{i 2}
$$

For the case $i=1$, the integration of (17) leads to

$$
\left(\langle\mu\rangle+\mu_{T}\right) \frac{\partial \bar{U}_{1}}{\partial x_{2}}=-f\left(x_{2}-h\right)
$$

At $x_{2}=0$ (where $\mu_{T}$ vanishes), this relation implies for the force $f$ the expression

$$
f=\frac{\mu_{w}}{h}\left[\frac{\partial \bar{U}_{1}}{\partial x_{2}}\right]_{x_{2}=0}=\frac{\tau_{w}}{h}
$$

The combination of (18) and (19) provides

$$
\left(\langle\mu\rangle+\mu_{T}\right) \frac{\partial \bar{U}_{1}}{\partial x_{2}}=-\tau_{w} \frac{x_{2}-h}{h}
$$

For the case $i=2$, the integration of (17) implies the relation

$$
\frac{2}{3}\langle\rho\rangle k+\langle\mathrm{p}\rangle=p_{w}
$$

The DNS data reveal that this consequence of modeling the turbulent fluxes by means of the Boussinesq approximation is well satisfied; see Fig. 6.

In analogy to Eq. (17), the temperature Eq. (13c) implies the balance

$$
0=\frac{\partial}{\partial x_{2}}\left(\frac{\langle\mu\rangle}{P r}+\frac{\mu_{T}}{P r_{t}}\right) \frac{\partial c_{p} \bar{T}}{\partial x_{2}}+\langle\rho\rangle \varepsilon+\langle\mu\rangle S^{2}
$$

Correspondingly, the integration of the mass fraction Eq. (13d) provides for the case considered

$$
\left(\frac{<\mu>}{S c}+\frac{\mu_{T}}{S c_{t}}\right) \frac{\partial \bar{m}}{\partial x_{2}}=\frac{\mu_{w}}{S c}\left[\frac{\partial \bar{m}}{\partial x_{2}}\right]_{w}
$$

With regard to the turbulent kinetic energy $k$, we derive from (15a) the balance

$$
0=\frac{1}{\langle\rho\rangle} \frac{\partial}{\partial x_{2}}\left(\frac{<\mu>}{P r_{k m}}+\frac{\mu_{T}}{P r_{k}}\right) \frac{\partial k}{\partial x_{2}}+C_{\mu} S^{2} \frac{k}{\omega}-k \omega
$$

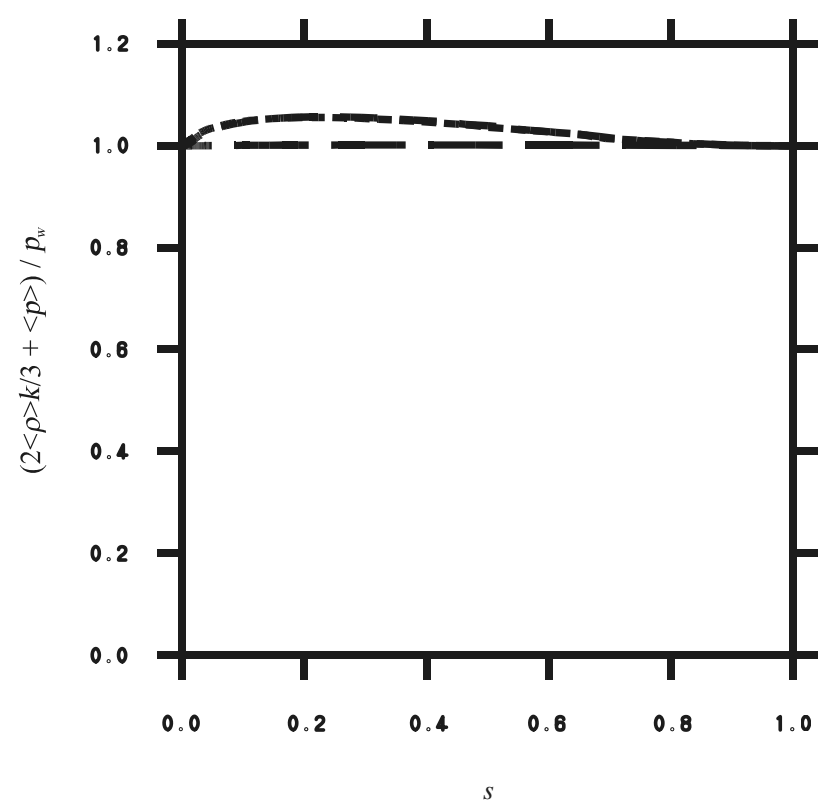

Fig. 6 Variation of $(2\langle\rho\rangle k / 3+\langle p\rangle) / p_{w}$ with $s$ [see relation (21)]. The same line coding is used as in Fig. 2.

Accordingly, (15b) provides the following balance for the turbulence frequency:

$$
0=\frac{1}{\langle\rho\rangle} \frac{\partial}{\partial x_{2}}\left(\frac{\langle\mu\rangle}{P r_{k m}}+\frac{\mu_{T}}{P r_{k}}\right) \frac{\partial \omega}{\partial x_{2}}-S_{\omega} \omega^{2}
$$

By adopting the DNS data presented in Sec. II, the use of the Eqs. (20) and (22-24) then allows to calculate all the turbulence model parameters $C_{\mu}, P r_{t}, S c_{t}, P r_{k m}, P r_{k}$, and $S_{\omega}$. For doing this, Eqs. (22) and (24) have to be integrated.

\section{Wall Damping Effects}

To prepare the calculation of model parameters let us consider first the effective turbulent viscosities in the mean velocity, temperature, mass fraction, and turbulent kinetic energy equations. By combining Eq. (20) for $\langle\mu\rangle+\mu_{T}$ with (8) for $s /\left|1-x_{2} / h\right|$, we obtain

$$
\frac{\mu_{w}}{\langle\mu\rangle+\mu_{T}}=\frac{s}{\left|1-x_{2} / h\right|}=0.02+0.98 s
$$

Figure 7 a shows that this linear function represents a very good approximation with regard to all the three DNS cases considered. This linear dependence describes the wall damping effect on the inverse effective normalized viscosity in a plausible way: the latter quantity has to be equal to 1 at $s=1$, and it has to decrease with increasing distance to the wall (with decreasing values of $s$ ).

As shown in Figs. 7b and 7c, the corresponding viscosities in the mean temperature and scalar equations may well be described by the same linear function,

$$
\frac{\mu_{w}}{\langle\mu\rangle+\mu_{T} / P r_{t}}=\frac{\mu_{w}}{\langle\mu\rangle+\mu_{T} / S c_{t}}=0.02+0.98 \mathrm{~s}
$$

The comparison with (26) leads to the conclusion that $P r_{t}=S c_{t}=1$ represent good approximations. This finding does not mean that there are no variations of $\mathrm{Pr}_{t}$ and $S c_{t}$; it means that the influence of variations of these numbers on the corresponding inverse effective viscosities is found to be negligible here. Regarding the temperature equation, it is worth noting that $\operatorname{Pr}_{t}$ was calculated on the basis of (22) by considering $P r=0.7$. The result for $P r_{t}$ was then used to plot the left-hand side of (27). To address the calculation of the turbulent viscosity in the turbulent kinetic energy equation, the left-hand side of (28) is plotted in Fig. 7d because it is well approximated by $0.02+0.98 s$ 


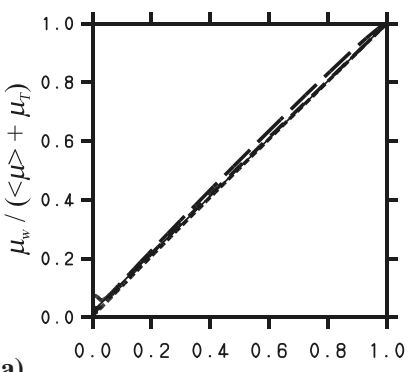

$S$

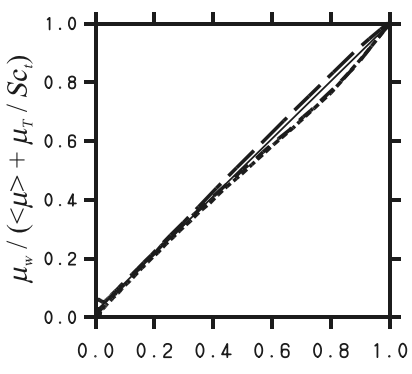

c)
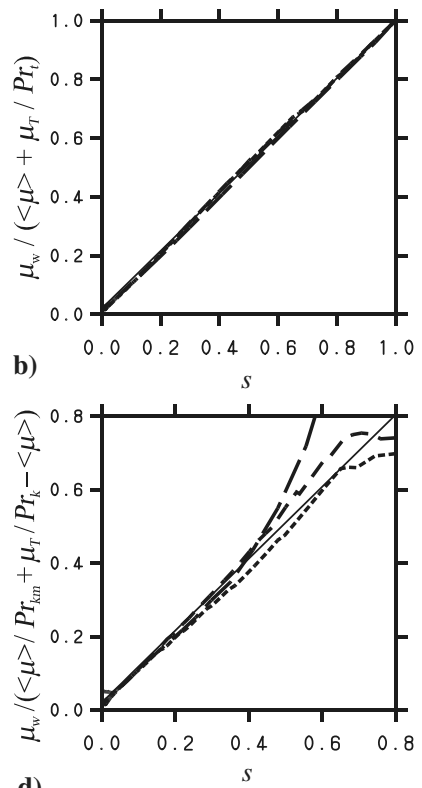

d)

Fig. 7 Variations of inverse effective normalized viscosities with $s$ [see relations (26-29)]. The same line coding is used as in Fig. 2. The linear function $0.02+\mathbf{0 . 9 8} \mathrm{s}$ is given in all figures for a comparison (solid line).
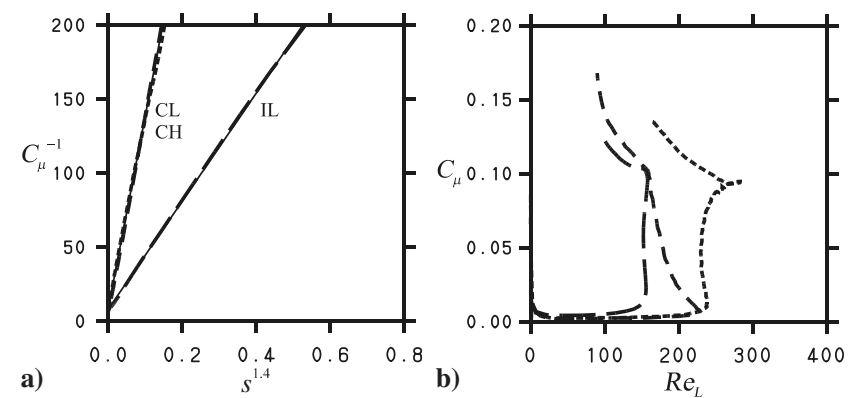

Fig. 8 Variations of $C_{\mu}^{-1}$ and $C_{\mu}$ with $s$ and $R e_{L}$, respectively (see the discussion in Sec. III.C). The same line coding is used as in Fig. 2. The functions (30) are given in a) for a comparison (solid lines).

$$
\frac{\mu_{w}}{\langle\mu\rangle / P r_{k m}+\mu_{T} / P r_{k}-\langle\mu\rangle}=0.02+0.98 s
$$

These data are only shown for $s<0.8$. The reason for that is given by the fact that unreasonable values were found at higher $s$; an appropriate calculation of derivatives in this range of huge variations of $\omega$ (see Fig. 3f) is, therefore, impossible. The combination of relations (26) and (28) then implies that

$$
2\langle\mu\rangle+\mu_{T}=\frac{\langle\mu\rangle}{P r_{k m}}+\frac{\mu_{T}}{P r_{k}}
$$

This relation is satisfied for all $\langle\mu\rangle$ and $\mu_{T}$ if $P r_{k}=1$ and $P r_{k m}=0.5$. The value $P r_{k}=1$ agrees well with $P r_{k}=S c_{t}=1$. The value $P r_{k m}=0.5$ is lower but comparable to the corresponding value $\operatorname{Pr}=0.7$ in the temperature equation.

The calculation of $C_{\mu}$ according to (20) combined with (14) for $\mu_{T}$ is shown in Fig. 8a. It is convenient to plot $C_{\mu}^{-1}$ because this quantity varies with $s^{1.4}$,

$$
\frac{1}{C_{\mu}}= \begin{cases}8+1300 s^{1.4} & \text { for CL and CH } \\ 8+365 s^{1.4} & \text { for IL }\end{cases}
$$

There is, therefore, a significant compressibility effect: $C_{\mu}$ decays with growing $s$ much faster for compressible flows, this means there is a stronger damping effects of walls. This behavior is again plausible due to the increase of mean mass density with decreasing wall distance in compressible flows. To understand the physics of $C_{\mu}$ variations in a clearer way, let us rewrite (30):

$$
\frac{\mathrm{d}}{\mathrm{d} \tau_{s}} \frac{1}{C_{\mu}}=-\frac{1.4}{\tau_{s}}\left(\frac{1}{C_{\mu}}-\frac{1}{C_{\mu}(\infty)}\right)
$$

Here, we introduced $\tau_{s}=1 / s$, which represents a characteristic time scale of mean flow variations. Further, we introduced $C_{\mu}(\infty)=1 / 8$, which is $C_{\mu}$ at $\tau_{s} \rightarrow \infty$ (this means at $s \rightarrow 0$ ). It is worth noting that $C_{\mu}(\infty)=0.12$ is in good agreement with values close to 0.1 applied usually for $C_{\mu}$ [3]. According to its definition (14), $1 / C_{\mu}=$ $\left(L k^{1 / 2}\right) / \nu_{T}$ may be considered as a turbulence Reynolds number. Equation (31) shows that this turbulence Reynolds number decreases with growing $\tau_{s}$ (with decreasing mean velocity gradients). Finally, $1 / C_{\mu}$ relaxes towards its equilibrium value $1 / C_{\mu}(\infty)$ at zero mean velocity gradients $(s=0)$. Relation (31) describes, therefore, a trend towards an equilibrium. The characteristic time scale $\tau_{s} / 1.4$ is equal for all the three cases considered. The difference between the incompressible case IL and compressible cases $\mathrm{CL}$ and $\mathrm{CH}$ [see Eq. (30)] is reflected in the wall condition at $\tau_{s}=1$ which is required for the solution of (31). This condition is given by $1 / C_{\mu}(1)=$ $(373 ; 1308 ; 1308)$ for the cases (IL; CL; CH), respectively.

The usual way to account for $C_{\mu}$ variations is to parameterize $C_{\mu}$ in terms of the turbulence Reynolds number $[3,16]$. To assess the suitability of such a parametrization, $C_{\mu}$ is plotted in Fig. $8 \mathrm{~b}$ against $R e_{L}$. This figure shows that the DNS data do not support a representation of $C_{\mu}$ as a unique function of $R e_{L}$ (see, for example, the case IL), which agrees with findings of Rodi and Mansour [25].

\section{The Turbulence Frequency Production}

The calculation of $S_{\omega}$ according to (25) is shown in Fig. 9. Figure 9a shows that a plot of $S_{\omega}$ against $s^{2}$ reveals linear variations of $S_{\omega}$ with $s^{2}$ for the cases CL and CH. However, the case IL cannot be described by a linear function of $s^{2}$. Instead, Fig. $9 \mathrm{c}$ shows that $S_{\omega}$ can be better described by a linear function of $s^{4}$ in this case. The functions presented in Figs. 9a and 9c are given by

$$
S_{\omega}= \begin{cases}-0.25+13.5 s^{2} & \text { for CL and CH } \\ -0.10+15.0 s^{4} & \text { for IL }\end{cases}
$$

We observe a significant compressibility effect, which may be explained in correspondence to the explanation of compressibility effects on $C_{\mu}$ : the increase of mean mass density with decreasing wall distance in compressible flows implies higher values of the normalized dissipation rate $S_{\omega}$. To understand the mechanism of turbulence frequency production, let us apply the same approach as used with regard to the analysis of $C_{\mu}$ variations. The corresponding rewriting of (32) reads

$$
\frac{\mathrm{d}}{\mathrm{d} \tau_{s}} S_{\omega}=-\frac{\beta_{\omega}}{\tau_{s}}\left[S_{\omega}-S_{\omega}(\infty)\right]
$$

The corresponding parameters and wall conditions are given in Table 3. The mechanism of turbulence frequency production is similar to the mechanism of $C_{\mu}$ variations. The source rate $S_{\omega}$ decreases with growing $\tau_{s}$ (with decreasing mean velocity gradients). Finally, $S_{\omega}$ relaxes towards its equilibrium value $S_{\omega}(\infty)$ at zero mean velocity gradients $(s=0)$. Thus, we observe again an evolution towards an equilibrium. A difference between (31) and (33) is given by the fact that compressibility decreases the characteristic mixing frequency $\beta_{\omega} / \tau_{s}$ in (33) by a factor of 2 , which may be again explained by the increase of mean mass density with decreasing wall distance.

$S_{\omega}$ is usually parameterized as a linear function of the productionto-dissipation ratio $P / \varepsilon$ of turbulent kinetic energy; see relation (16). To analyze the relation between these two quantities, $S_{\omega}$ is plotted against $P / \varepsilon$ in Fig. 9e. This figure supports the view that $S_{\omega}$ varies linearly with $P / \varepsilon$ only for values $S_{\omega}>0.5$ (which corresponds to the near wall region before $P / \varepsilon$ reaches its maximum; see Fig. 5c). Therefore, a correlation between $S_{\omega}$ and $P / \varepsilon$ that applies to all the flow does not exist. 

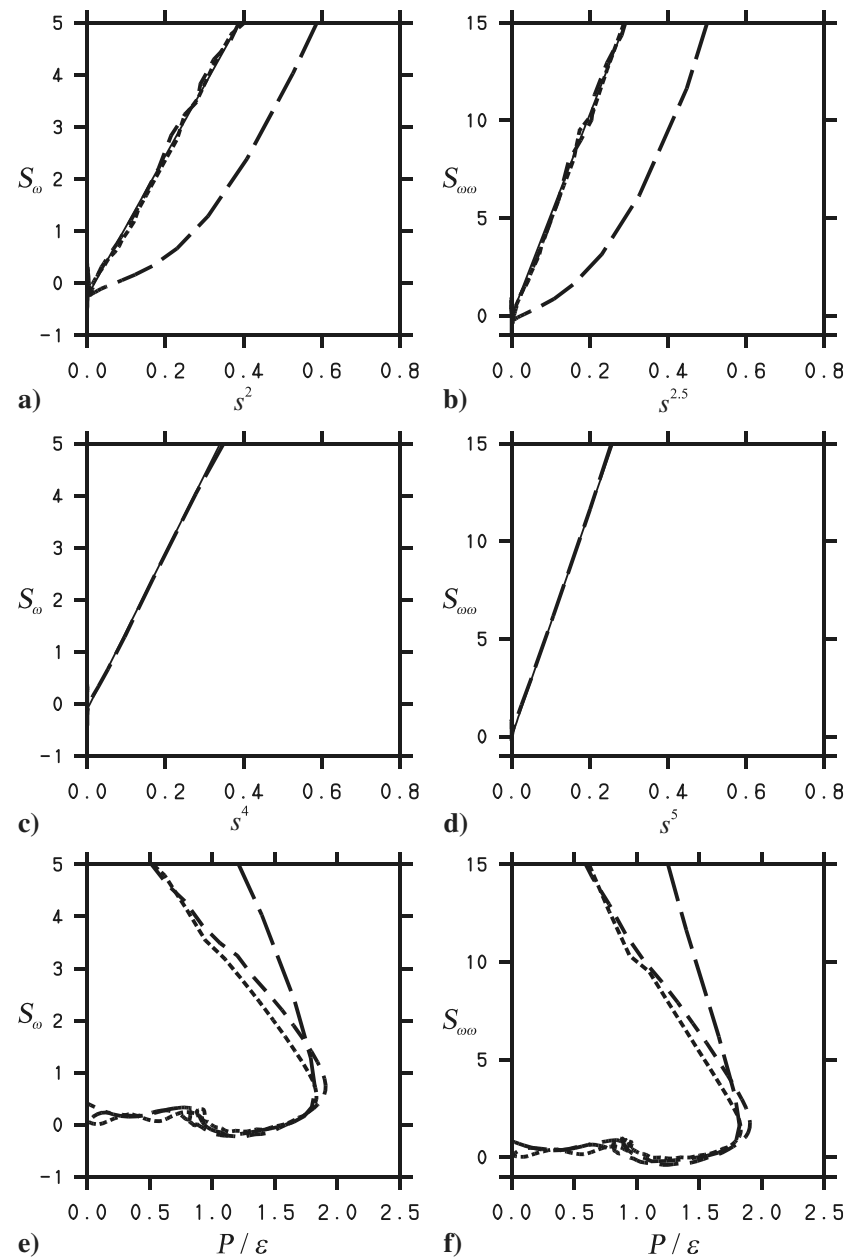

Fig. 9 The figures a), c), and e) show the variations of $S_{\omega}$ with $s^{2}, s^{4}$, and $P / \varepsilon$, respectively: c) shows only the case IL (see the discussion in Sec. III. D). The figures b), d), and f) show the variations of $S_{\omega \omega}$ with $s^{2.5}, s^{5}$, and $P / \varepsilon$, respectively: $d)$ shows only the case $I L$. The same line coding is used as in Fig. 2. The corresponding approximations (32) for $S_{\omega}$ and (35) for $S_{\omega \omega}$ are presented in a), c) and b), d) for a comparison (solid lines).

It is of interest to compare these findings obtained for the $k-\omega$ turbulence model with corresponding features of other two-equation turbulence models. As discussed in detail by Wilcox [16], one may combine the turbulent kinetic energy equation with a variety of other scale determining equations: for example, with equations for the squared turbulence frequency, dissipation rate $\varepsilon$, turbulence time scale $\tau$, and eddy length scale $L$. By introducing the source rates $S_{\omega \omega}$, $S_{\varepsilon}, S_{\tau}$, and $S_{L}$, these model equations are given in analogy to Eq. (25) by

$$
\begin{aligned}
0 & =\frac{1}{\langle\rho\rangle} \frac{\partial}{\partial x_{2}}\left(\frac{\langle\mu\rangle}{P r_{k m}}+\frac{\mu_{T}}{P r_{k}}\right) \frac{\partial \omega^{2}}{\partial x_{2}}-S_{\omega \omega} \omega^{3} \\
0 & =\frac{1}{\langle\rho\rangle} \frac{\partial}{\partial x_{2}}\left(\frac{\langle\mu\rangle}{P r_{k m}}+\frac{\mu_{T}}{P r_{k}}\right) \frac{\partial \varepsilon}{\partial x_{2}}-S_{\varepsilon} \omega \varepsilon \\
0 & =\frac{1}{\langle\rho\rangle} \frac{\partial}{\partial x_{2}}\left(\frac{\langle\mu\rangle}{P r_{k m}}+\frac{\mu_{T}}{P r_{k}}\right) \frac{\partial \tau}{\partial x_{2}}-S_{\tau} \\
0 & =\frac{1}{\langle\rho\rangle} \frac{\partial}{\partial x_{2}}\left(\frac{\langle\mu\rangle}{P r_{k m}}+\frac{\mu_{T}}{P r_{k}}\right) \frac{\partial L}{\partial x_{2}}-S_{L} k^{1 / 2}
\end{aligned}
$$

The features of the $k-\omega^{2}$ turbulence model are compared with those of the $k-\omega$ turbulence model in Fig. 9. This figure reveals that the behavior of the $k-\omega^{2}$ turbulence model is very similar to the behavior of the $k-\omega$ turbulence model. Figure $9 \mathrm{~b}$ shows that $S_{\omega \omega}$ grows linearly with $s^{2.5}$ for the cases $\mathrm{CL}$ and $\mathrm{CH}$, but the case IL cannot be described by a linear function of $s^{2.5}$. Instead, Fig. 9d shows that $S_{\omega \omega}$ can be better described by a linear function of $s^{5}$ in
Table 3 Parameters and wall conditions at $\tau_{s}=1$ related to the $S_{\omega}$ and $S_{\omega \omega}$ Eqs. (33) and (36)

\begin{tabular}{lccllc}
\hline \hline Case & $\beta_{\omega}$ & $S_{\omega}(\infty)$ & $S_{\omega}(1)$ & $\beta_{\omega \omega}$ & $S_{\omega \omega}(1)$ \\
\hline $\mathrm{IL}$ & 4 & -0.10 & 14.9 & 5 & 18.5 \\
$\mathrm{CL}$ & 2 & -0.25 & 13.25 & 2.5 & 16.5 \\
$\mathrm{CH}$ & 2 & -0.25 & 13.25 & 2.5 & 16.5 \\
\hline \hline
\end{tabular}

this case. Appropriate approximations for the variation of $S_{\omega \omega}$ are then given by

$$
S_{\omega \omega}= \begin{cases}16.5 s^{2.5} & \text { for CL and CH } \\ 18.5 s^{5} & \text { for IL }\end{cases}
$$

These relations may be rewritten in correspondence to the structure of Eq. (33):

$$
\frac{\mathrm{d}}{\mathrm{d} \tau_{s}} S_{\omega \omega}=-\frac{\beta_{\omega \omega}}{\tau_{s}} S_{\omega \omega}
$$

The corresponding $\beta_{\omega \omega}$ and wall values are given in Table 3. Obviously, the discussion of Eq. (33) also applies to (36). Regarding the relation between $S_{\omega \omega}$ with the production-to-dissipation ratio $P / \varepsilon$ of turbulent kinetic energy one finds very similar features as for $S_{\omega}$; a correlation between $S_{\omega \omega}$ and $P / \varepsilon$ that applies to all the flow does not exist.

Corresponding calculations of $S_{\varepsilon}, S_{\tau}$, and $S_{L}$ as functions of $s$ and $P / \varepsilon$ are shown in Fig. 10 (as in Fig. 7d, these plots are only shown for $s<0.8$ because an appropriate calculation of derivatives for higher $s$ values was found to be impossible). These figures reveal two
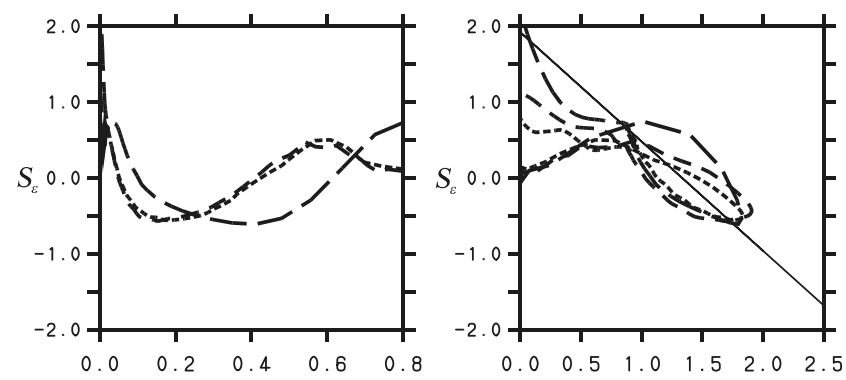

a)

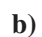

b)

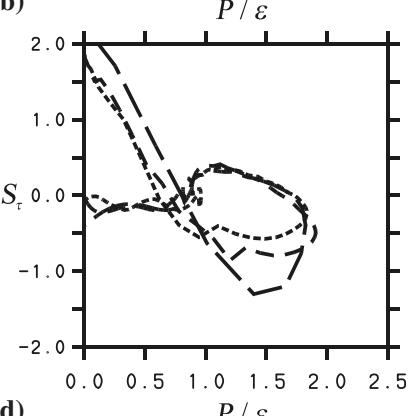

c)

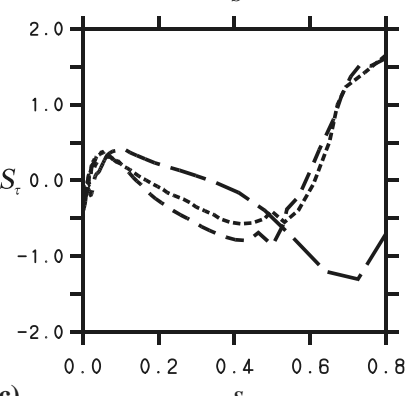

d)
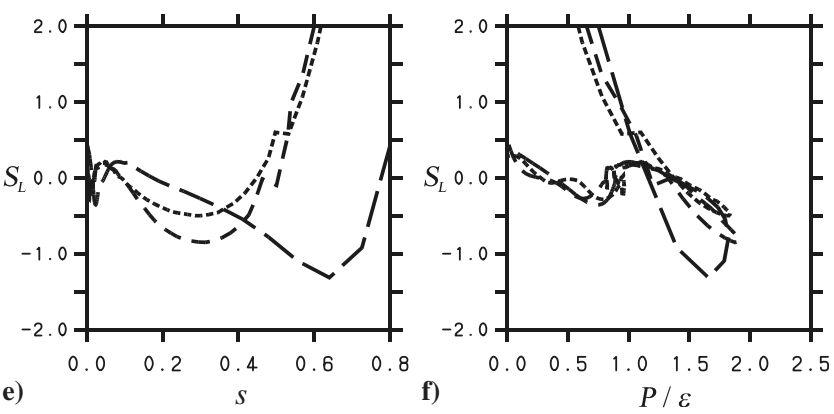

Fig. 10 Variations of $S_{\varepsilon}, S_{\tau}$, and $S_{L}$ with $s$ and $P / \varepsilon$ (see the discussion in Sec. III.D). The same line coding is used as in Fig. 2. The standard model $1.92-1.44 P / \varepsilon$ for the source rate $S_{\varepsilon}$ in the dissipation rate equation $[3,16]$ is shown in b) for a comparison (solid line). 
common features: relatively simple and unique parameterizations of these source rates as functions of $s$ or $P / \varepsilon$ are unavailable, and there are significant compressibility effects. The standard model $1.92-$ $1.44 P / \varepsilon$ for the source rate $S_{\varepsilon}$ in the dissipation rate equation $[3,16]$ is shown in Fig. 10b for a comparison. It may be seen that this model can only indicate the general trend of $S_{\varepsilon}$ variations (with a significant inaccuracy).

\section{Summary and Concluding Remarks}

The answers obtained to the questions discussed in the introduction (the turbulence frequency production mechanism, wall damping effects on turbulence model parameters, and the relevance of compressibility effects) will be summarized in this section.

The characteristic time scale $\tau$ of turbulent velocity fluctuations has to be provided to close both deterministic RANS and stochastic PDF methods. Usually, $\tau$ is calculated in terms of transport equations for the turbulence frequency $\omega=1 / \tau$ or dissipation rate $\varepsilon=k / \tau$. The most important terms in such transport equations are the source rates, as, for example, $S_{\omega}$ and $S_{\varepsilon}$ in turbulence frequency and dissipation rate equations, respectively. In analogy to the corresponding source rate $1-P / \varepsilon$ in the turbulent kinetic energy equation, the source rates $S_{\omega}$ and $S_{\varepsilon}$ are usually assumed to be linear functions of $P / \varepsilon$. It was shown here that there is only very limited support available for such an assumption with regard to $S_{\omega}$ : a corresponding linear relation between $S_{\omega}$ and $P / \varepsilon$ can only be found in the near-wall region before $P / \varepsilon$ reaches its maximum. Thus, a correlation between $S_{\omega}$ and $P / \varepsilon$ that applies to all the flow does not exist. With regard to $S_{\varepsilon}$, it was shown that the standard model $1.92-1.44 P / \varepsilon$ for the source rate $S_{\varepsilon}$ in the dissipation rate equation can only indicate the general trend of $S_{\varepsilon}$ variations (with a significant inaccuracy).

In contrast to these findings it was shown that accurate parameterizations for $S_{\omega}$ and $S_{\omega \omega}$ as functions of the scaling parameter $s$ can be derived (see Fig. 9). The mechanism of $S_{\omega}$ and $S_{\omega \omega}$ variations can be explained by means of the Eqs. (33) and (36): the source rates $S_{\omega}$ and $S_{\omega \omega}$ evolve towards their equilibrium values $S_{\omega}(\infty)$ and zero, respectively. This evolution is controlled by the characteristic time scale $\tau_{s}$ of mean flow changes. It was also shown that other formulations of scale determining equations are less appropriate (see Fig. 10).

The second question considered in the introduction concerned the explanation of variations of turbulence model parameters for wallbounded flows. To explain variations of turbulence model parameters one presently applies two concepts $[3,16]$ : the scaling of coefficients in terms of the turbulence Reynolds number, or their scaling with normalized wall distances. Regarding the use of $R e_{L}$, it was demonstrated here that there is no support available for such scalings (which agrees with conclusions derived by Rodi and Mansour ). Scaling concepts based on wall distances do not provide turbulence models which are invariant under the Galilean transformation. Such scaling concepts do not have a broad range of applicability; one needs different (inner and outer) scalings for different flow regions, and inner scaling turned out to be inapplicable to compressible flow [31]. The use of such concepts may also become very problematic with regard to flows in complex geometries or multicomponent reacting flows. Thus, there is a need for the development of wall effect scalings that are independent of wall parameters [49].

In contrast to the problems described in the preceding paragraph, it was shown in detail in Sec. II.B that the use of $s$ as scaling parameter offers significant advantages. The main reason for that is the reference to a local flow state that is independent of wall properties (the latter is very helpful, for example, with regard to simulations of flows in complex geometries or multicomponent reacting flows). Regarding the turbulence Schmidt number and Prandtl numbers in temperature and turbulent kinetic energy equations, it was found that $S c_{t}=P r_{t}=P r_{k}=1$ represent good assumptions. It is worth noting that the analysis presented here revealed the need to involve a molecular Prandtl number $P r_{k m}=0.5$ in the turbulent kinetic energy equation. The calculation of $C_{\mu}$ showed that $1 / C_{\mu}$ scales with $s^{1.4}$.
The mechanism of $C_{\mu}$ variations is explained by Eq. (31); in correspondence to $S_{\omega}$ and $S_{\omega \omega}$ variations one observes a trend towards the equilibrium value of $C_{\mu}$.

An important question concerns the influence of compressibility effects on the turbulence characteristics and models considered here. It is worth emphasizing that investigations of such effects are not only relevant to compressible flows; studies of the effects of mass density and temperature variations considered in this way are also important to chemically reacting flows.

As shown in Secs. II.B-II.D, compressibility effects are relevant to the flows considered: the characteristic length and time scales of turbulent eddies and production and dissipation of turbulent kinetic energy are clearly affected by compressibility. Regarding the relevance of compressibility effects to turbulence model parameters one observes that such effects do not modify turbulence parameterizations in the mean velocity, temperature, mass fraction, and turbulent kinetic energy equations: $\left(\langle\mu\rangle+\mu_{T}\right) / \mu_{w}, P r_{t}, S c_{t}$, $P r_{k}$, and $P r_{k m}$ are unaffected. In accord with these findings one finds that the evolution (31) of $C_{\mu}$ is also unaffected; compressibility modifies $C_{\mu}$ only via the wall condition $C_{\mu}(1)$ related to Eq. (31). It is worth emphasizing that there is no need to account for such effects if expression (26) is applied for the normalized effective inverse turbulent viscosity. Compressibility effects are found to be relevant regarding the evolution of the source rates $S_{\omega}$ and $S_{\omega \omega}$ in corresponding scale determining equations (compressibility decreases the characteristic mixing frequencies $\beta_{\omega} / \tau_{s}$ and $\beta_{\omega \omega} / \tau_{s}$ in Eqs. (33) and (36) by a factor of 2). This variation should be taken into account in turbulence models.

\section{Acknowledgments}

This work was supported by the Deutsche Forschungsgemeinschaft (SFB 255 [TP A2], Transfer Project 42, and FR 478/20-2) and FLUENT Germany (M. Braun) as part of a program related to the development of improved numerical methods for turbulent combustion. I am very thankful to H. Foysi for providing the DNS data applied and many helpful suggestions. Support of J. Kreuzinger regarding the use of DNS data is gratefully acknowledged. I am very thankful to R. Friedrich and W. Rodi for valuable comments. Many thanks also to the referees for their helpful advice.

\section{References}

[1] Baldyga, J., and Bourne, J. R., Turbulent Mixing and Chemical Reactions, John Wiley \& Sons, New York, 1999.

[2] Pope, S. B., "A Perspective on Turbulence Modeling," Modeling Complex Turbulent Flows, edited by M. D. Salas, J. N. Hefner, and L. Sakell, Kluwer, Dordrecht, The Netherlands, 1999, pp. 53-67.

[3] Pope, S. B., Turbulent Flows, Cambridge Univ. Press, Cambridge, England, U.K., 2000.

[4] Peters, N., Turbulent Combustion, Cambridge Univ. Press, Cambridge, England, U.K., 2001.

[5] Durbin, P. A., and Petterson, B. A., Statistical Theory and Modeling for Turbulent Flows, John Wiley \& Sons, New York, 2001.

[6] Fox, R. O., Computational Models for Turbulent Reacting Flows, Cambridge Series in Chemical Engineering, Cambridge Univ. Press, Cambridge, England, U.K., 2003.

[7] Heinz, S., Statistical Mechanics of Turbulent Flows, Springer-Verlag, Berlin, 2003.

[8] Givi, P., "A Review of Modern Developments in Large Eddy Simulation of Turbulent Reacting Flows," DNS/LES-Progress and Challenges, edited by C. Liu, L. Sakell, and R. Herklotz, Greyden, Columbus, OH, 2001, pp. 81-92.

[9] Givi, P., "Filtered Density Function for Subgrid Scale Modeling of Turbulent Combustion," AIAA Journal, Vol. 44, No. 1, 2006, pp. 16 23.

[10] Colucci, P. J., Jaberi, F. A., Givi, P., and Pope, S. B., "Filtered Density Function for Large Eddy Simulations of Turbulent Reactive Flows," Physics of Fluids, Vol. 10, No. 2, 1998, pp. 499-515.

[11] Jaberi, F. A., Colucci, P. J., James, S., Givi, P., and Pope, S. B., "Filtered Mass Density Function for Large-Eddy Simulation of Turbulent Reacting Flows," Journal of Fluid Mechanics, Vol. 401, 1999, pp. 85121. 
[12] Gicquel, L. Y. M., Givi, P., Jaberi, F. A., and Pope, S. B. "Velocity Filtered Density Function for Large Eddy Simulation of Turbulent Flows," Physics of Fluids, Vol. 14, No. 3, 2002, pp. 11961213.

[13] Sheikhi, M. R. H., Drozda, T. G., Givi, P., and Pope, S. B., "VelocityScalar Filtered Density Function for Large-Eddy Simulation of Turbulent Flows," Physics of Fluids, Vol. 15, No. 8, 2003, pp. 23212337.

[14] Lumley, J. L., "Computational Modeling of Turbulent Flows," Advances in Applied Mechanics, Vol. 18, 1978, pp. 123-175.

[15] Kolmogorov, A. N., "The Equations of Turbulent Motion in an Incompressible Fluid," (in Russian), Physics, Vol. 6, Izvestia Academy of Sciences, USSR, pp. 56-58.

[16] Wilcox, D. C., Turbulence Modeling for CFD, 2nd ed., DCW Industries, La Cañada, CA, 1998.

[17] Hanjalić, K., "Advanced Turbulence Closure Models: A View of Current Status and Future Prospects," International Journal of Heat and Fluid Flow, Vol. 15, No. 3, 1994, pp. 178-203.

[18] Lumley, J. L., and Khajeh Nouri, B., "Computational Modelling of Turbulent Transport," Advances in Geophysics, Vol. 18A, 1974, pp. 169-192.

[19] Yakhot, V., and Orszag, S. A., "Renormalization Group Analysis of Turbulence, Part 1. Basic Theory," Journal of Scientific Computing, Vol. 1, No. 1, 1986, pp. 3-51.

[20] Yakhot, V., Orszag, S. A., Thangam, S., Gatski, T. B., and Speziale, C. G., "Development of Turbulence Model for Shear Flows by Double Expansion Technique," Physics of Fluids A, Vol. 4, No. 7, 1992, pp. $1510-1520$.

[21] Smith, L. M., and Reynolds, W. C., "On the Yakhot-Orszag Renormalization Group Method for Deriving Turbulence Statistics and Models," Physics of Fluids A, Vol. 4, No. 2, 1992, pp. 364-390.

[22] Yakhot, V., and Smith, L. M., "The Renormalization Group, the $\varepsilon$ Expansion and Derivation of Turbulence Models," Journal of Scientific Computing, Vol. 7, No. 1, 1992, pp. 35-61.

[23] Smith, L. M., and Woodruff, S. L., "Renormalization-Group Analysis of Turbulence," Annual Review of Fluid Mechanics, Vol. 30, 1998, pp. 275-310.

[24] Launder, B. E., "Phenomenological Modeling: Present and Future," Wither Turbulence? Turbulence at the Crossroads, edited by J. L. Lumley, Springer-Verlag, Berlin, 1990, pp. 439-485.

[25] Rodi, W., and Mansour, N. N., "Low Reynolds Number $k-\varepsilon$ Modelling with the Aid of Direct Numerical Simulation Data," Journal of Fluid Mechanics, Vol. 250, 1993, pp. 509-529.

[26] Blaisdell, G. A., Mansour, N. N., and Reynolds, W. C., "Compressibility Effects on the Growth and Structure of Homogeneous Turbulent Shear Flow," Journal of Fluid Mechanics, Vol. 256, 1993, pp. 443-485.

[27] Chassaing, P., Antonia, R. A., Anselmet, F., Joly, L., and Sarkar, S., Variable Density Fluid Turbulence, Fluid Mechanics and its Applications, Vol. 69, Kluwer Academic Publishers, Dordrecht, the Netherlands, 2002.

[28] Coleman, G. N., Kim, J., and Moser, R. D., "A Numerical Study of Turbulent Supersonic Isothermal-Wall Channel Flow," Journal of Fluid Mechanics, Vol. 305, 1995, pp. 159-183.

[29] Foysi, H., and Friedrich, R., "DNS of Passive Scalar Transport in Turbulent Supersonic Channel Flow," Proceedings of the 3rd International Symposium on Turbulence and Shear Flow Phenomena, edited by N. Kasagi, J. K. Eaton, R. Friedrich, J. A. C. Humphrey, M. A. Leschziner, and T. Miyauchi, Vol. 3, Sendai, Japan, June 2003, pp. 1121-1126.

[30] Foysi, H., Sarkar, S., and Friedrich, R., "On Reynolds Stress Anisotropy in Compressible Channel Flow," Proceedings of the 3rd International Symposium on Turbulence and Shear Flow Phenomena, edited by N. Kasagi, J. K. Eaton, R. Friedrich, J. A. C. Humphrey, M. A. Leschziner, and T. Miyauchi, Vol. 3, Sendai, Japan, June 2003, pp. 1103-1108.
[31] Foysi, H., Sarkar, S., and Friedrich, R., "Compressibility Effects and Turbulence Scalings in Supersonic Channel Flow," Journal of Fluid Mechanics, Vol. 509, 2004, pp. 207-216.

[32] Foysi, H., "Transport passiver Skalare in wandgebundener und isotroper kompressibler Turbulenz,," (in German), Ph.D. Dissertation, Technical University of Munich (Fachgebiet Strömungsmechanik), Munich, Feb. 2005.

[33] Freund, J. B., "Compressibility Effects in a Turbulent Annular Mixing Layer," Ph.D. Thesis, Mechanical Engineering Dept., Stanford Univ., Stanford, CA, 1997.

[34] Freund, J. B., Lele, S. K., and Moin, P., "Compressibility Effects in a Turbulent Annular Mixing Layer. Part 1. Turbulence and Growth Rate," Journal of Fluid Mechanics, Vol. 421, 2000, pp. 229 267.

[35] Freund, J. B., Moin, P., and Lele, S. K., "Compressibility Effects in a Turbulent Annular Mixing Layer. Part 2. Mixing of a Passive Scalar," Journal of Fluid Mechanics, Vol. 421, 2000, pp. 269-292.

[36] Friedrich, R., "Modelling of Turbulence in Compressible Flows," Transition, Turbulence and Combustion Modelling, edited by A. Hanifi, P. H. Alfredsson, A. V. Johansson, and D. S. Henningson, Kluwer Academic Publishers (ERCOFTAC Series), Dordrecht, the Netherlands, 1999, pp. 243-348.

[37] Heinz, S., "A Model for the Reduction of the Turbulent Energy Redistribution by Compressibility," Physics of Fluids, Vol. 15, No. 11, 2003, pp. 3580-3583.

[38] Huang, P. G., Coleman, G. N., and Bradshaw, P., "Compressible Turbulent Channel Flows: DNS Results and Modelling," Journal of Fluid Mechanics, Vol. 305, 1995, pp. 185-218.

[39] Lechner, R., Sesterhenn, J., and Friedrich, R., "Turbulent Supersonic Channel Flow," Journal of Turbulence, Vol. 2, 2001, pp. 1-25.

[40] Lele, S. K., "Compressibility Effects on Turbulence," Annual Review of Fluid Mechanics, Vol. 26, 1994, pp. 211-154.

[41] Pantano, C., and Sarkar, S., "A Study of Compressibility Effects in the High-Speed Turbulent Shear Layer Using Direct Simulation," Journal of Fluid Mechanics, Vol. 451, 2002, pp. 329-371.

[42] Sarkar, S., "The Stabilizing Effect of Compressibility in Turbulent Shear Flow," Journal of Fluid Mechanics, Vol. 282, 1995, pp. 163186.

[43] Sesterhenn, J., "A Characteristic-Type Formulation of the NavierStokes Equations for High-Order Upwind Schemes," Computers \& Fluids, Vol. 30, No. 1, 2001, pp. 37-67.

[44] Adams, N. A., and Shariff, K., "A High-Resolution Hybrid CompactENO Scheme for Shock-Turbulence Interaction Problems," Journal of Computational Physics, Vol. 127, No. 1, 1996, pp. 27-51.

[45] Lele, S. K., "Compact Finite Difference Schemes with Spectral-Like Resolution," Journal of Computational Physics, Vol. 103, No. 1, 1992, pp. 16-42.

[46] Williamson, J. H., "Low-Storage Runge-Kutta Schemes," Journal of Computational Physics, Vol. 35, No. 1, 1980, pp. 48-56.

[47] Kim, J., Moin, P., and Moser, R. D., "Turbulence Statistics in Fully Developed Channel Flow at Low Reynolds Number," Journal of Fluid Mechanics, Vol. 177, 1987, pp. 133-166.

[48] Moser, R. D., Kim, J., and Mansour, N. N., "Direct Numerical Simulation of Turbulent Channel Flow up to $R e_{\tau}=590$," Physics of Fluids, Vol. 11, No. 4, 1999, pp. 943-945.

[49] Launder, B. E., and Li, S.-P., "On the Elimination of Wall-Topography Parameters from Second-Moment Closure," Physics of Fluids, Vol. 6, No. 2, 1994, pp. 999-1006.

[50] Heinz, S., "On the Kolmogorov Constant in Stochastic Turbulence Models," Physics of Fluids, Vol. 14, No. 11, 2002, pp. 4095-4098.

[51] FLUENT 6.3 User's Guide, FLUENT, Lebanon, NH, 2006. 